\title{
Excited State Dynamics Affected by Switching of Hydrogen-Bond Network in Hydrated Aminopyrazine Clusters
}

Yuji Yamada, Yuji Goto, Yuki Fukuda, Hiroumi Ohba, and Yoshinori Nibu*

Department of Chemistry, Faculty of Science, Fukuoka University, Jonan-ku, Fukuoka 814-0180, Japan

Table of Contents

Figure S1 Expanded LIF and UV-UV HB spectra of $\mathrm{APz}-\left(\mathrm{H}_{2} \mathrm{O}\right)_{2}$

Figure S2 Optimized geometries of the most stable five isomers for $\mathrm{APz}-\left(\mathrm{H}_{2} \mathrm{O}\right)_{4}$

Figure S3 Simulated IR spectra of the most stable five isomers for $\mathrm{APz}-\left(\mathrm{H}_{2} \mathrm{O}\right)_{4}$

Figure S4 Dependence of simulated IR on calculation level

Figure S5 Comparison of LIF spectra of hydrated APz, 2AP, and 3AP clusters

Figure S6 Optimized cluster geometries at the high calculation level

Table S1 TD-DFT calculation for $\mathrm{APz}-\left(\mathrm{H}_{2} \mathrm{O}\right)_{n}(n=0-4)$

Figure S7 Kohn-Sham orbitals for APz- $\left(\mathrm{H}_{2} \mathrm{O}\right)_{n}(n=0-4)$ 


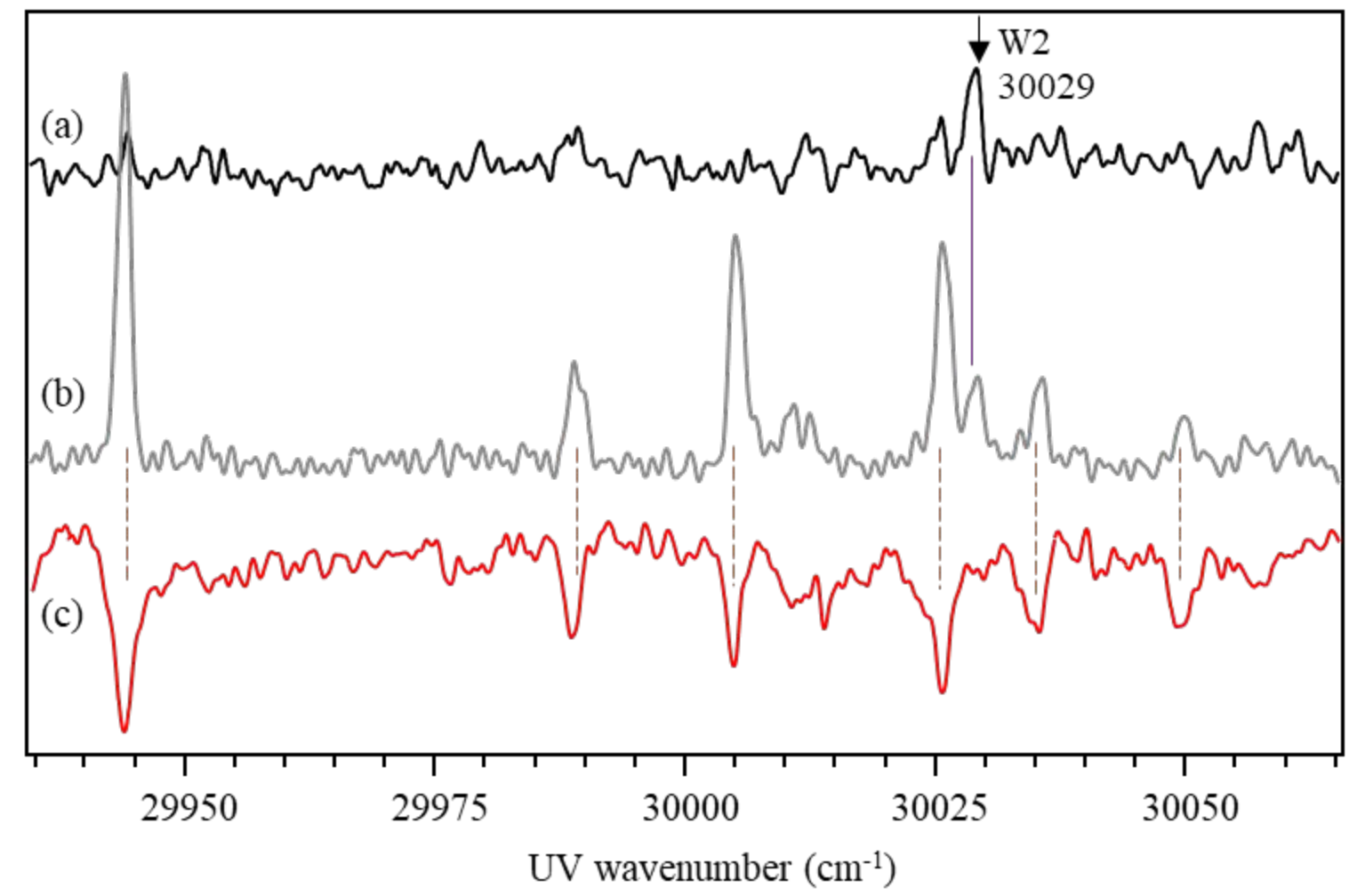

Figure S1. Expanded LIF spectra around the origin band of $\mathrm{APz}^{-}\left(\mathrm{H}_{2} \mathrm{O}\right)_{2}$, denoted by $\mathrm{W} 2$, in the different condition with a water reservoir cooled to (a) 278 and (b) $288 \mathrm{~K}$, and UV-UV HB spectrum measured by fixing the probe UV frequencies to $29851 \mathrm{~cm}^{-1}$, that is the origin band of $\mathrm{APz}^{-}\left(\mathrm{H}_{2} \mathrm{O}\right)_{3}$. 
(a)

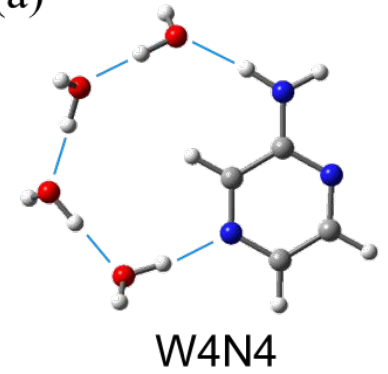

$0.0(0.0)$ (b)

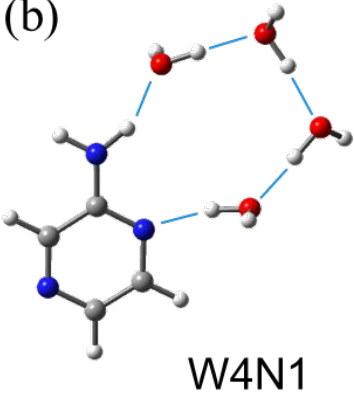

$+3.1(+3.8)$ (c)
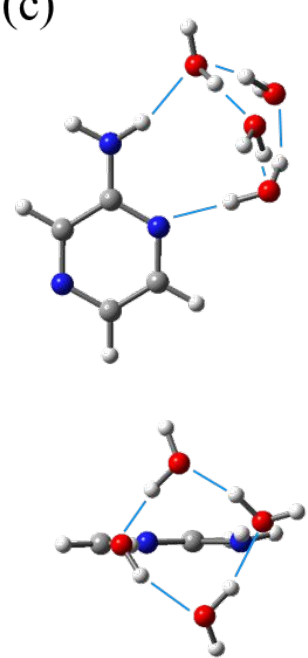

W4_3+1a

$+3.5(+3.1)$ (d)
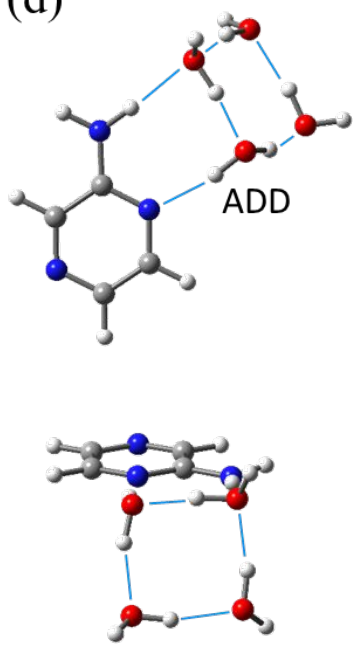

W4_2+2a

$+2.0(+1.9)$ (e)
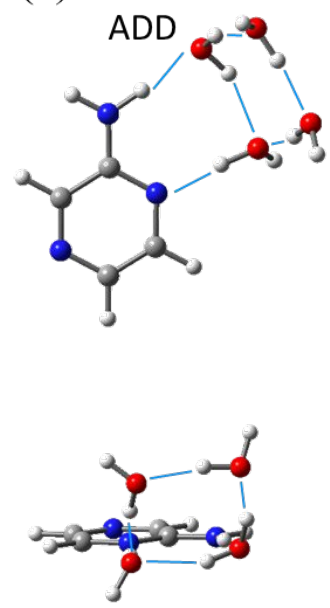

W4_2+2b

$+2.8(+2.4)$

Figure S2. Optimized geometries of the most stable five isomers for $\mathrm{APz}-\left(\mathrm{H}_{2} \mathrm{O}\right)_{4}$ and their relative stabilization energies $(\mathrm{kcal} / \mathrm{mol})$, calculated at the B3LYP/6-311++G(d,p) level. The values in parenthesis indicate those calculated at the MP2 level. The lower in the figure $(\mathrm{c}-\mathrm{d})$ displays the side view. 


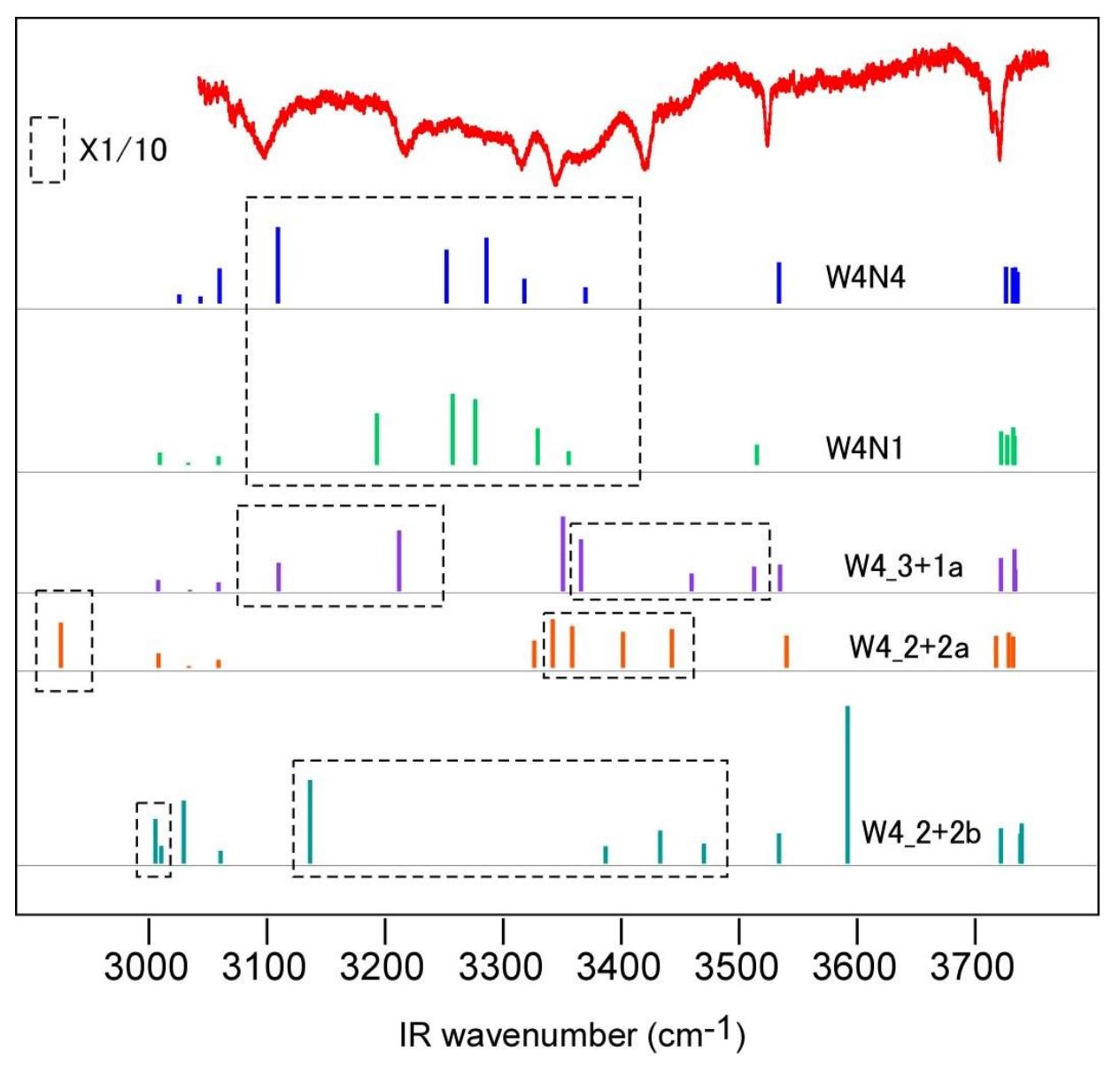

Figure S3. Simulated IR spectra of the the most stable five isomers for $\mathrm{APz}-\left(\mathrm{H}_{2} \mathrm{O}\right)_{4}$ described in Figure S2. The calculation level was B3LYP/6-311++G(d,p). The observed spectrum was also shown for comparison. Bands surrounded by broken lines in the figure mean the band intensity scaled by $1 / 10$ times. 


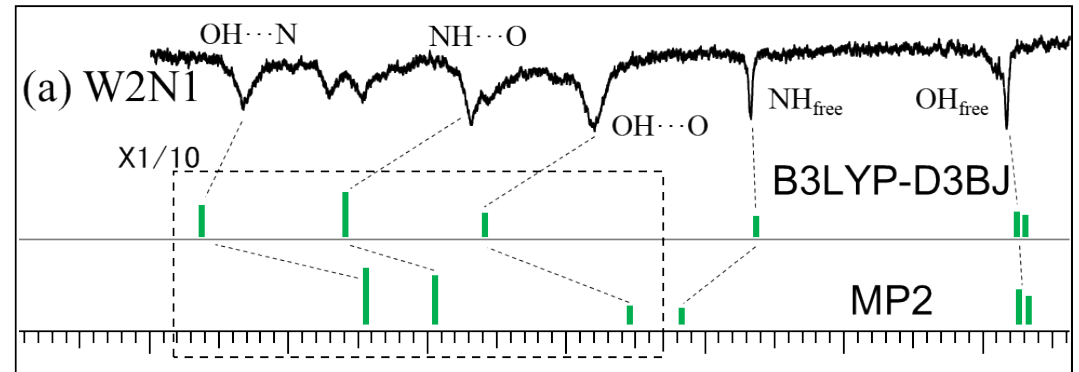

(b) W3N4
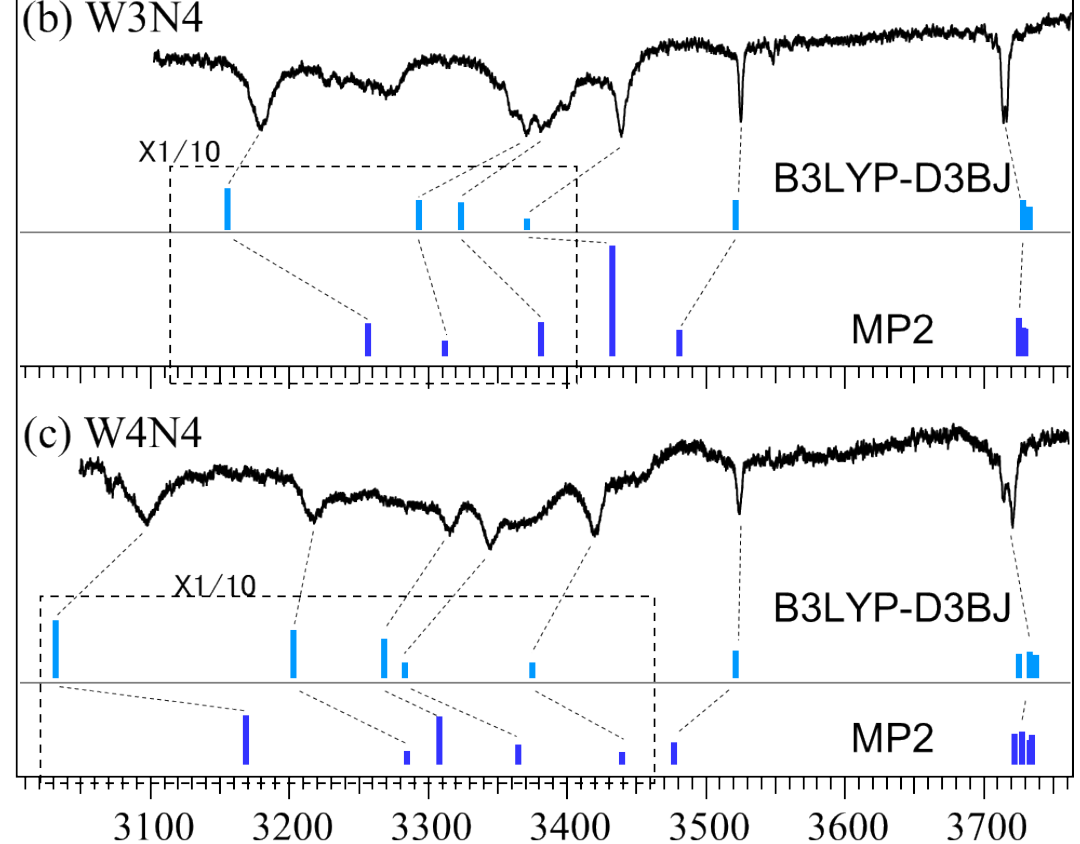

Figure S4. Vibrational spectral dependence on calculation levels for the most stable isomers of (a) W2N1, (b) W3N4, and (c) W4N4 for the APz clusters. Basis sets of 6-311++G(d,p) were used in all levels. Scaling factors were adopted to be 0.9596 in B3LYP-D3BJ and 0.9440 in MP2. Bands surrounded by broken lines in the figure mean the band intensity scaled by $1 / 10$ times in order to make it easy to compare. They exhibit almost similar spectral features with B3LYP one in Figure 3. 


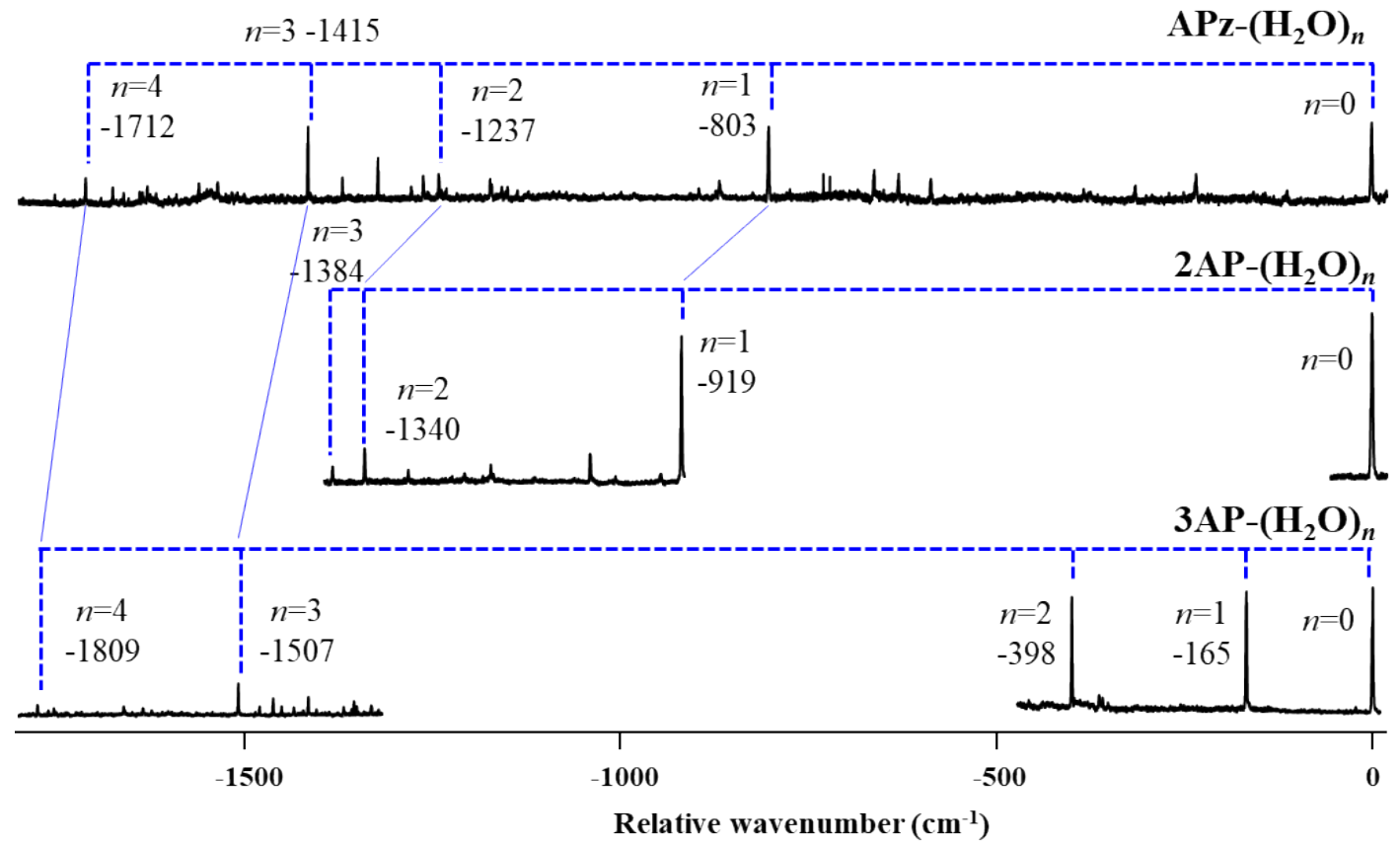

Figure S5. Comparison of LIF spectra of hydrated APz, 2AP, and 3AP clusters as a function of the relative frequency from the origin band of monomer.

(a)

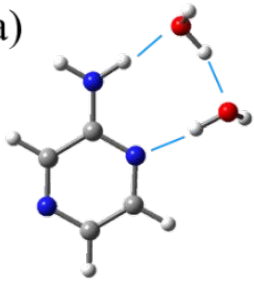

W2N1

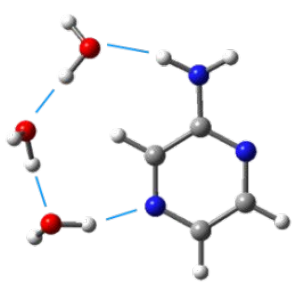

W3N4

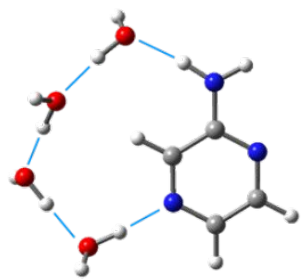

W4N4

(b)

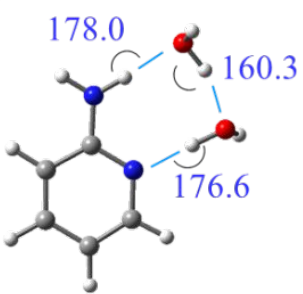

2AP-W2

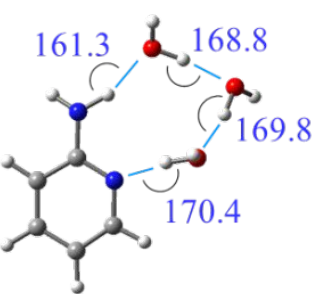

$2 \mathrm{AP}-\mathrm{W} 3_{\mathrm{ch}}$

Figure 6S. Optimized geometries of the global minima for (a) $\mathrm{APz}-\left(\mathrm{H}_{2} \mathrm{O}\right)_{n}(n=2-4)$, and (b) $2 \mathrm{AP}-\left(\mathrm{H}_{2} \mathrm{O}\right)_{n}(n=2,3)$, obtained using B3LYP-D3BJ/6-311++G(d,p). The inserted values mean the $\mathrm{H}$-bond angles in degree. 
Table S1. Vertical transition energies $\left(\mathrm{E}_{\mathrm{vert}}\right)$ in $\mathrm{eV}$ and configuration interaction (CI) coefficient for the $\mathrm{S}_{n}$ and $\mathrm{T}_{n}(n=1-3)$ excited states of the global minima of $\mathrm{APz}^{-}\left(\mathrm{H}_{2} \mathrm{O}\right)_{n}(n=0-4)$ calculated using the TD-DFT calculation with the CAM-B3LYP/6-311++G(d,p) level.

\begin{tabular}{llllllllll}
\hline & \multicolumn{1}{c}{$\mathrm{S}_{1}$} & \multicolumn{1}{c}{$\mathrm{S}_{2}$} \\
\cline { 2 - 10 } & $\mathrm{E}_{\text {vert }}$ & \multicolumn{2}{l}{ CI coefficient $^{a}$} & $\mathrm{E}_{\text {vert }}$ & \multicolumn{2}{l}{ CI coefficient $^{a}$} & \multicolumn{2}{l}{$\mathrm{E}_{\text {vert }}$} & CI coefficient $^{a}$ \\
\hline W0 & 4.4258 & $24->26$ & 0.69771 & 4.6583 & $23->29$ & 0.11770 & 5.5118 & $24->29^{b}$ & 0.64604 \\
& & & & & $25->26$ & 0.69072 & & & \\
W1 & 4.4682 & $29->31$ & 0.69533 & 4.5088 & $30->31$ & 0.69251 & 5.6483 & $30->32$ & 0.61280 \\
W2N1 & 4.4371 & $34->36$ & 0.26438 & 4.4894 & $34->36$ & 0.64555 & 5.6129 & $35->37$ & 0.57740 \\
& & $35->36$ & 0.64461 & & $35->36$ & -0.26324 & & & \\
W3N4 & 4.3909 & $40->41$ & 0.69423 & 4.5799 & $39->41$ & 0.69824 & 5.6822 & $40->42$ & -0.33071 \\
& & & & & & & & $40->44$ & -0.22207 \\
W4N4 & 4.3322 & $45->46$ & 0.69643 & 4.5857 & $44->46$ & 0.69962 & 5.6335 & $45->47$ & 0.51468 \\
\hline
\end{tabular}

\begin{tabular}{|c|c|c|c|c|c|c|c|c|c|}
\hline \multirow[b]{3}{*}{ W0 } & \multirow{3}{*}{$\begin{array}{l}\mathrm{T}_{1} \\
\frac{\mathrm{E}_{\text {vert }}}{3.3312}\end{array}$} & \multirow{2}{*}{\multicolumn{2}{|c|}{ CI coefficient ${ }^{a}$}} & \multicolumn{3}{|l|}{$\mathrm{T}_{2}$} & \multicolumn{3}{|l|}{$\mathrm{T}_{3}$} \\
\hline & & & & $E_{\text {vert }}$ & \multicolumn{2}{|c|}{ CI coefficient ${ }^{a}$} & $E_{\text {vert }}$ & \multicolumn{2}{|c|}{ CI coefficient ${ }^{a}$} \\
\hline & & $25->26$ & 0.69321 & 3.6512 & $23->26$ & -0.27159 & 3.6768 & $24->26$ & 0.68344 \\
\hline & & & & & $25->29$ & 0.59033 & & & \\
\hline \multirow[t]{3}{*}{ W1 } & 3.1382 & $30->31$ & 0.69556 & 3.6321 & $27->31$ & -0.25282 & 3.7155 & $29->31$ & 0.68020 \\
\hline & & & & & $30->34$ & 0.54317 & & & \\
\hline & & & & & $30->35$ & -0.29049 & & & \\
\hline \multirow[t]{2}{*}{ W2N1 } & 3.0516 & $35->36$ & 0.69588 & 3.6228 & $32->36$ & 0.20668 & 3.7296 & $34->36$ & 0.67635 \\
\hline & & & & & $35->40$ & 0.57668 & & & \\
\hline \multirow[t]{2}{*}{ W3N4 } & 2.9890 & $40->41$ & 0.69670 & 3.6093 & $38->41$ & 0.24989 & 3.9122 & $39->41$ & 0.69106 \\
\hline & & & & & $40->45$ & 0.59922 & & & \\
\hline \multirow[t]{2}{*}{ W4N4 } & 2.9193 & $45->46$ & 0.69684 & 3.6033 & $43->46$ & -0.22185 & 3.9268 & $44->46$ & 0.69175 \\
\hline & & & & & $45->50$ & 0.58533 & & & \\
\hline
\end{tabular}

${ }^{a}$ Two numbers point out the occupied and unoccupied Kohn-Sham orbitals for a single excitation, which are displayed in Figure S7.

${ }^{b}$ This transition implies the second $\pi \pi^{*}$ state, while the $\pi \sigma^{*}$ state illustrated in Figure 7 in the article corresponds to $\mathrm{S}_{4}$ having the transition energy of $5.5382 \mathrm{eV}$. 


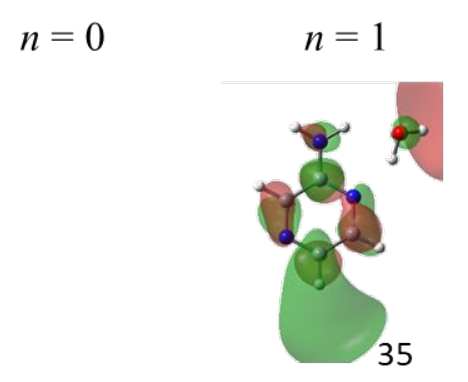

$$
n=2
$$

$$
n=3
$$

$$
n=4
$$
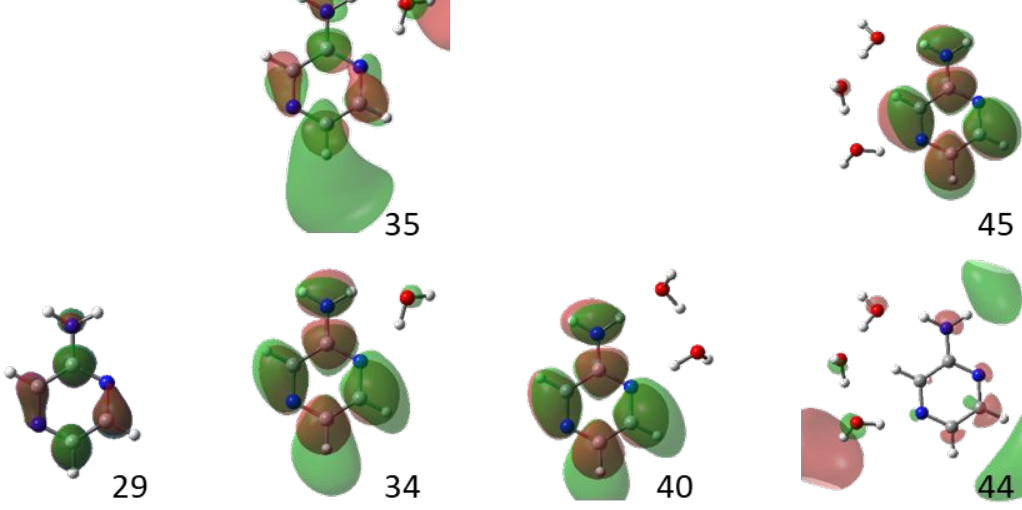

45
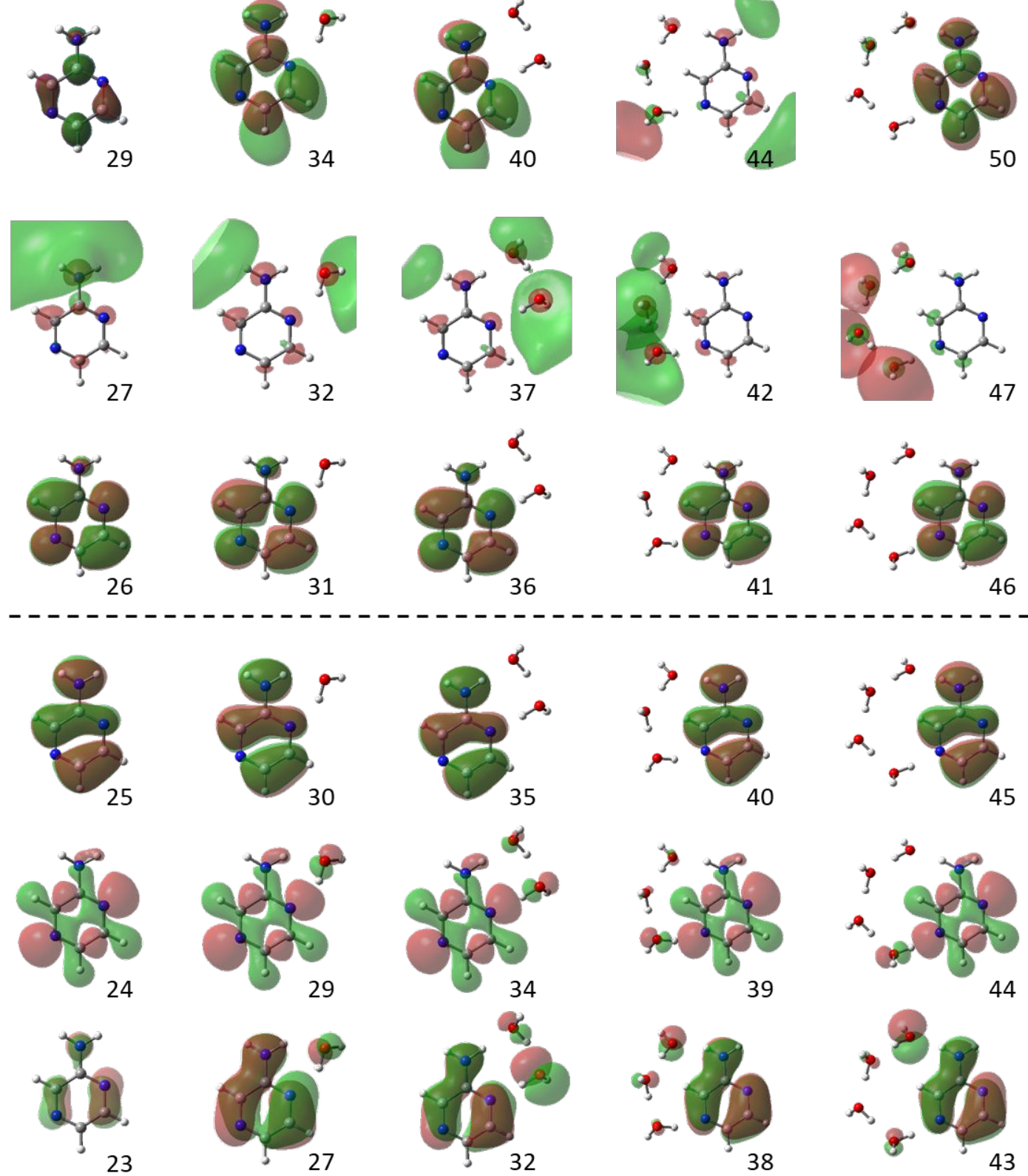

Figure S7. Kohn-Sham orbitals mainly related to the $\mathrm{S}_{n}-\mathrm{S}_{0}$ transitions $(n=1-3)$ for the global minima of $\mathrm{APz}-\left(\mathrm{H}_{2} \mathrm{O}\right)_{n}(n=0-4)$ calculated at the CAM-B3LYP/6-311++G(d,p) level, corresponding to a density isosurface value of 0.05 . 